\title{
Novel open cavity design for rotating mode rf pulse compressors
}

\author{
Xiaowei Wu $\odot^{*}$ and Alexej Grudiev \\ European Organization for Nuclear Research (CERN), 1211 Geneva 23, Switzerland
}

(Received 14 July 2021; accepted 14 October 2021; published 9 November 2021)

\begin{abstract}
A new $X$-band high-power rotating mode SLAC energy doubler (SLED)-type rf pulse compressor is proposed. It is based on a novel cavity type, a single open bowl-shaped energy storage cavity with highquality factor and compact size, which is coupled to the waveguide using a compact rotating mode launcher. The novel cavity type is applied to the rf pulse compression system of the main linac rf module of the klystron-based option of the Compact Linear Collider. Quasispherical rotating modes of $\mathrm{TE}_{1,2,4}$ and $\mathrm{TE}_{1,2,13}$ are proposed for the correction cavity and storage cavity of the if pulse compression system, respectively. The storage cavity working at $\mathrm{TE}_{1,2,13}$ has a quality factor above 240000 and a diameter less than $33 \mathrm{~cm}$. The design of the pulse compressor and in particular of the high-Q cavity will be presented in detail.
\end{abstract}

DOI: 10.1103/PhysRevAccelBeams.24.112001

\section{INTRODUCTION}

As an alternative to the original two beam Compact Linear Collider (CLIC) concept, the klystron-based CLIC option is considered for the $380 \mathrm{GeV}$ initial energy stage [1]. An $X$-band rf pulse compressor with a correction cavity chain was selected as a baseline option for the klystronbased CLIC rf module [2-4]. Rf compression obtains high peak power in exchange for reduced rf pulse length. Pulse compressors have been applied and operated for many decades in many facilities, such as SLAC National Accelerator Laboratory, Swiss Free Electron Laser, SPring8 Angstrom Compact free electron LAser (SACLA), Pohang Accelerator Laboratory X-ray Free Electron Laser (PAL-XFEL), and Shanghai soft X-ray FreeElectron Laser facility (SXFEL) [5-13]. The first rf pulse compressor named SLAC energy doubler (SLED) was developed by Perry Wilson, David Farkas, Harry Hoag, and Gregory Loew at SLAC [14]. The key components of a SLED system include a $3 \mathrm{~dB}$ coupler with two $90^{\circ}$ apart divided power ports and two high-intrinsic quality factor $\left(Q_{0}\right)$ energy storage cavities. The concept was further developed during era of $X$-band linear colliders: VLEPP/ NLC/JLC [15-17]. The several ideas used in this paper were introduced at that time. Open cavity with two rotating modes was invented by Vladimir Balakin and realized by Igor Syratchev of BINP as barrel open cavity [18]. Deference with the current design is that the mode launcher

\footnotetext{
*xiaowei.wu@cern.ch
}

Published by the American Physical Society under the terms of the Creative Commons Attribution 4.0 International license. Further distribution of this work must maintain attribution to the author(s) and the published article's title, journal citation, and DOI. was in the form of a directional coupler. Correction cavities were invented by Sergey Kazakov, then from KEK, to simulate frequency response of a SLED-II delay line $[2,19]$. Using a polarizer is a more recent development, where a spherical cavity fed by $H$-plane polarizer was invented by Sami Tantawi and developed into a practical device by Juwen Wang, both of SLAC [20-22]. Two such SLEDs, each with a $Q_{0}$ of $9.5 \times 10^{4}$, are now in use at linac coherent light source. The version with $H$-plane polarizer propagated around the world and many were built in China and elsewhere [23-26]. An $X$-band corrugated cavity with a $Q_{0}$ of $1.15 \times 10^{5}$ was also developed based on the concept of polarizer [27-29]. Author of this paper, Alexej Grudiev contributed into the art by inventing an $E$-plane polarizer, which could substitute the $H$-plane polarizer in a SLED with rotating mode cavity [30].

The parameters of the CLIC accelerating structure had been reevaluated to improve the accelerator performance [31]. Additionally, the pulse compressor design adopted to the new structure parameters was optimized based on the klystron output [32]. It has been suggested previously that a correction cavity chain with a $Q_{0}$ of $6 \times 10^{4}$ used in conjunction with a storage cavity with a $Q_{0}$ of $2.4 \times 10^{5}$ could increase the achievable power gain by $7.5 \%$ compared to the initial design, with the initial design having a gain factor of 3.48 and the new scheme having a gain factor of 3.74. The correction cavity chain is used to produce flattop and increase the efficiency [3]. The scheme, pulse shape, and transmission spectrum of the CLIC pulse compression system are illustrated in Fig. 1. Phase-toamplitude modulation by using two klystrons could be applied to eliminate the ripples of the output pulse in Fig. 1(b) [3]. The correction cavities and storage cavity with higher $Q_{0}$ could increase the achievable power gain. To implement such a scheme, however, new storage cavity 

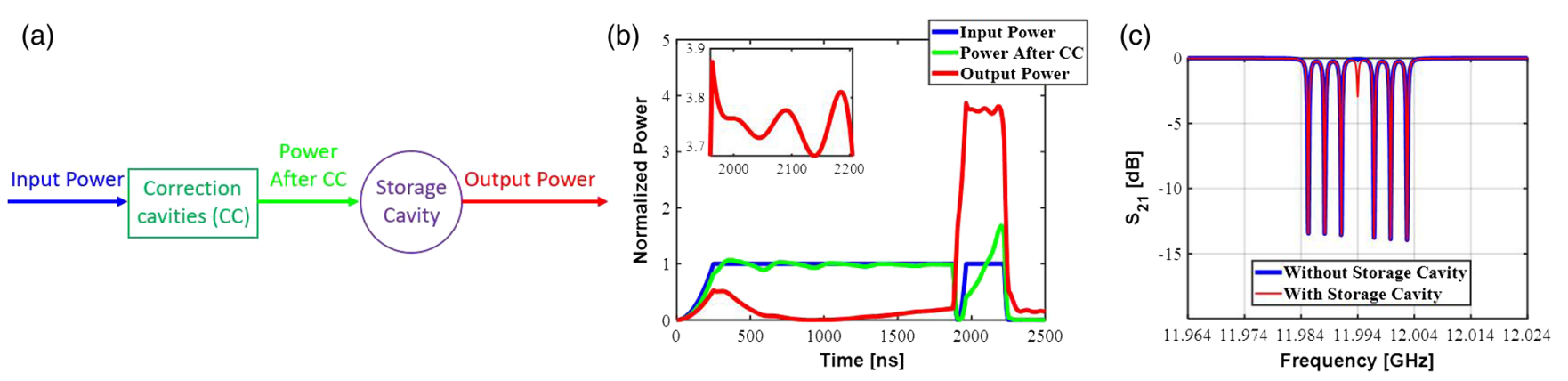

FIG. 1. (a) Scheme, (b) pulse shape, and (c) transmission spectrum of the pulse compression system of klystron-based $380 \mathrm{GeV}$ CLIC option. The blue, green, and red curves in (b) are the input power, power after the correction cavity chain, and output power, respectively. The inset of (b) is given to show an enlarged view of output power. The $Q_{0}$ of storage cavity and coupling factor are $2.4 \times 10^{5}$ and 6 . The $Q_{0}$ of correction cavity and coupling factor are $6.0 \times 10^{4}$ and 1.48 . Phase-to-amplitude modulation by using two klystrons could be applied to eliminate the ripples of the output pulse [3]. The blue and red curves in (c) are the transmission spectra of the correction cavity chain and the whole system. The frequency difference between two nearby peaks is $2.9 \mathrm{MHz}$, which corresponds to the pulse length of $344 \mathrm{~ns}$.

and correction cavity chain which meet these criteria must be designed. A novel compact open bowl-shaped energy storage cavity with high $Q_{0}$ is proposed to meet the requirements for both the correction cavity chain and the storage cavity of CLIC. This novel cavity type with rotating mode could also be applied to other pulse compression systems. This paper describes its principle, design, and technical advances.

\section{BOWL-SHAPED OPEN CAVITY}

The novel open cavity has a bowl shape, as shown in Fig. 2. The electrical field of the working mode in the top area of the cavity is very small. Thus the top of the cavity can be kept open. The cavity works in a quasispherical rotating mode of $\mathrm{TE}_{1,2, i}$ which is a dipole mode. The radial

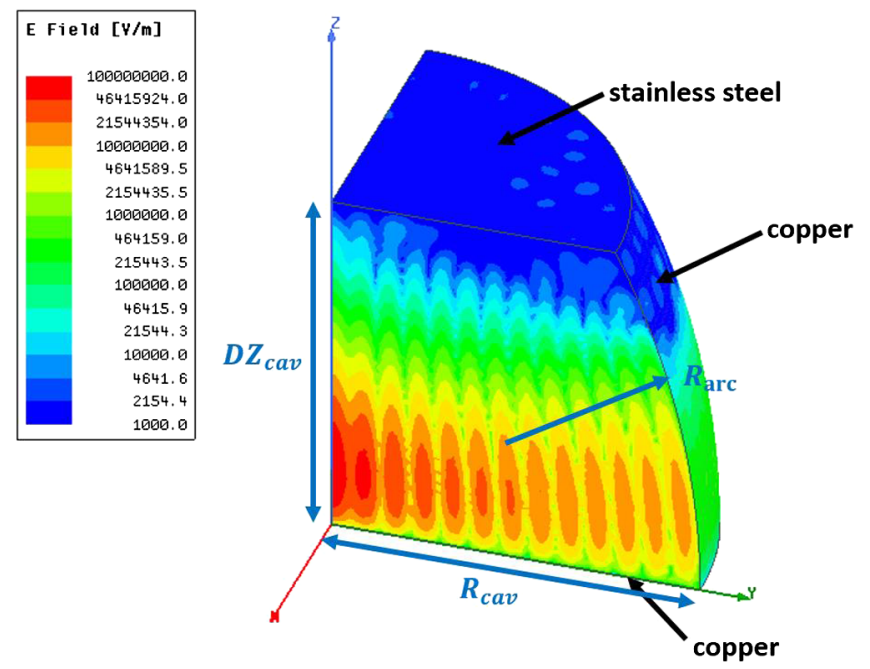

FIG. 2. Electrical field of the bowl-shaped open cavity operating at $\mathrm{TE}_{1,2,13}$ at $11.994 \mathrm{GHz}$. One quarter model of the cavity with a storage energy of $1 \mathrm{~J}$ was simulated by the eigenmode solver of high-frequency structure simulator (HFSS) [33]. The electrical field is in logarithmic scale. index $i$ is dominated by the cavity radius $\left(R_{\text {cav }}\right)$. The working mode shown in Fig. 2 is $\mathrm{TE}_{1,2,13}$. The $Q_{0}$ of $\mathrm{TE}_{1,2,13}$ at $11.994 \mathrm{GHz}$ is around $2.4 \times 10^{5}$. Principally, a larger $R_{\text {cav }}$ contributes to a higher radial index which results in a higher $Q_{0}$. However, more parasitic modes will appear in the larger cavity. The parasitic modes may have high field on the top area which results in high loss in the open boundary. The coupling from parasitic modes to working modes will impact the performance of the pulse compressor. The frequency separation between working mode and parasitic modes should be kept as large as possible. Otherwise absorption materials such as silicon carbide need to be added to damp such modes. $D Z_{\text {cav }}$ and $R_{\text {arc }}$ are used to optimize the $Q_{0}$ of the working mode and the mode separation between working mode and parasitic modes.

The open boundary will also help us to suppress many parasitic modes. The cavity can be easily machined by lathe due to its symmetric and open shape. No brazing is needed for the cavity body manufacturing. This can reduce the cost of the fabrication and increase the fabrication accuracy. The top of the cavity is connected to the stainless steel flange with pumping port which will make it easy to pump.

\section{CORRECTION CAVITY DESIGN}

\section{A. Cavity design}

The $Q_{0}$ of the correction cavity chain is suggested to be $6 \times 10^{4}$ from Ref. [32]. $\mathrm{TE}_{1,2,4}$ mode is selected for the bowl-shaped open cavity design with the $R_{\text {cav }}$ around $50 \mathrm{~mm}$. The $Q_{0}$ is around 75000 which is larger than the requirement. As mentioned in the previous chapter, coupling to the parasitic modes is one of the critical issues in the design. A single cavity model was created to calculate the stainless steel quality factor $\left(Q_{\mathrm{ss}}\right)$ and the mode spectrum, as shown in Fig. 3. $Q_{\mathrm{ss}}$ is defined as $\frac{2 \pi f W}{P_{\mathrm{ss}}}$, where $f$ is the resonant frequency, $W$ is the stored energy, and $P_{\mathrm{ss}}$ is the power loss in the stainless steel. To reduce the 


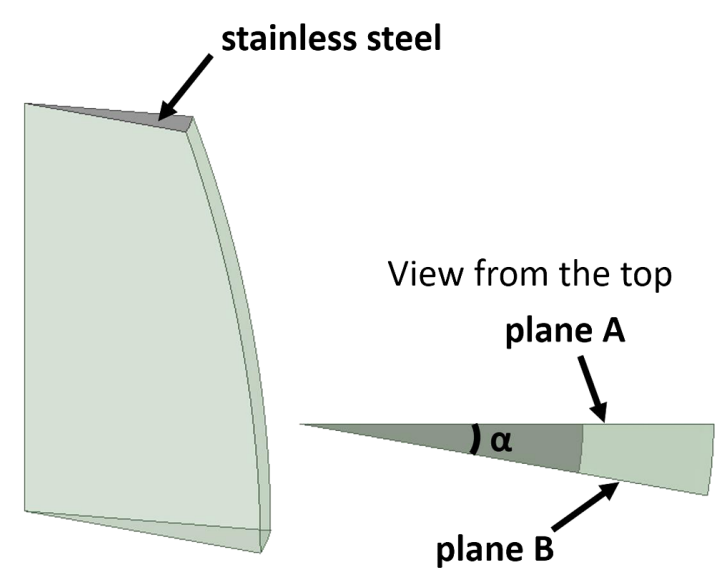

FIG. 3. The azimuthal slice of the cavity with a geometric angle of $\alpha$ degrees used in HFSS for mode spectrum calculation. The top of the model was set as stainless steel boundary while the rf phase advance $\left(\theta_{\mathrm{ph}}\right)$ between planes $\mathrm{A}$ and $\mathrm{B}$ was set to be the same as the geometric angle $(\alpha)$. $\alpha$ was set to 8 degrees in the simulation.

simulation time, an azimuthal slice of the cavity with a geometric angle of $\alpha$ degrees was used in HFSS simulation, as shown in Fig. 3. A periodic boundary was then set at each side of the slice, and the azimuthal rf phase advance between them, $\theta_{\mathrm{ph}}$, was set to the geometric angle ( $\alpha$ degrees). The phase advance between the boundary walls determines the azimuthal index of the modes which will be simulated, and relates the geometric angle, $\alpha$, to the azimuthal index, $\mathrm{m}$, as

$$
m=\theta_{\mathrm{ph}} / \alpha
$$

Thus, by setting the phase advance to $\alpha$, only modes with an azimuthal index of one, $\mathrm{TE}_{1, n, p}$ modes, are simulated. No other mode will be excited in case of perfect rotational symmetric coupling. The top of the model was set as a stainless steel boundary.

The mode spectrum for different $R_{\text {arc }}$ is shown in Fig. 4. The middle line with high $Q_{\mathrm{ss}}$ is the working mode of $\mathrm{TE}_{1,2,4}$ while the other lower $Q_{\mathrm{ss}}$ points represent the parasitic modes. $R_{\text {arc }}=300 \mathrm{~mm}$ is selected for the correction cavity design as it has a large mode separation from the nearest two parasitic modes and has a high $Q_{\mathrm{ss}}$ value.

\section{B. Coupler design}

Though the optimized cavity shape has a large frequency separation between working mode and parasitic modes, the coupling to the parasitic modes may still increase the field at the open area of the cavity after adding the coupling iris. Therefore, the loss in the open boundary area needs to be checked. The coupling iris model is presented in Fig. 5. The top of the cavity model with coupling iris and circular waveguide is set to stainless steel boundary. $\mathrm{TE}_{11}$ rotating mode propagates in the circular waveguide and excites $\mathrm{TE}_{1,2,4}$ mode in the open cavity via the coupling iris. Note that the coupler will only excite modes with an azimuthal index of one in case of perfect rotational symmetry. A quantity named $R_{\text {loss }}$ is defined as the ratio between the loss in the stainless steel and that in the copper of the model. The coupling iris is optimized to get the required $Q_{\text {ext }}$ and minimum $R_{\text {loss }}$. The minimum $R_{\text {loss }}$ of $0.05 \%$ is obtained when $R_{\text {iris }}$ is $4.44 \mathrm{~mm}$.

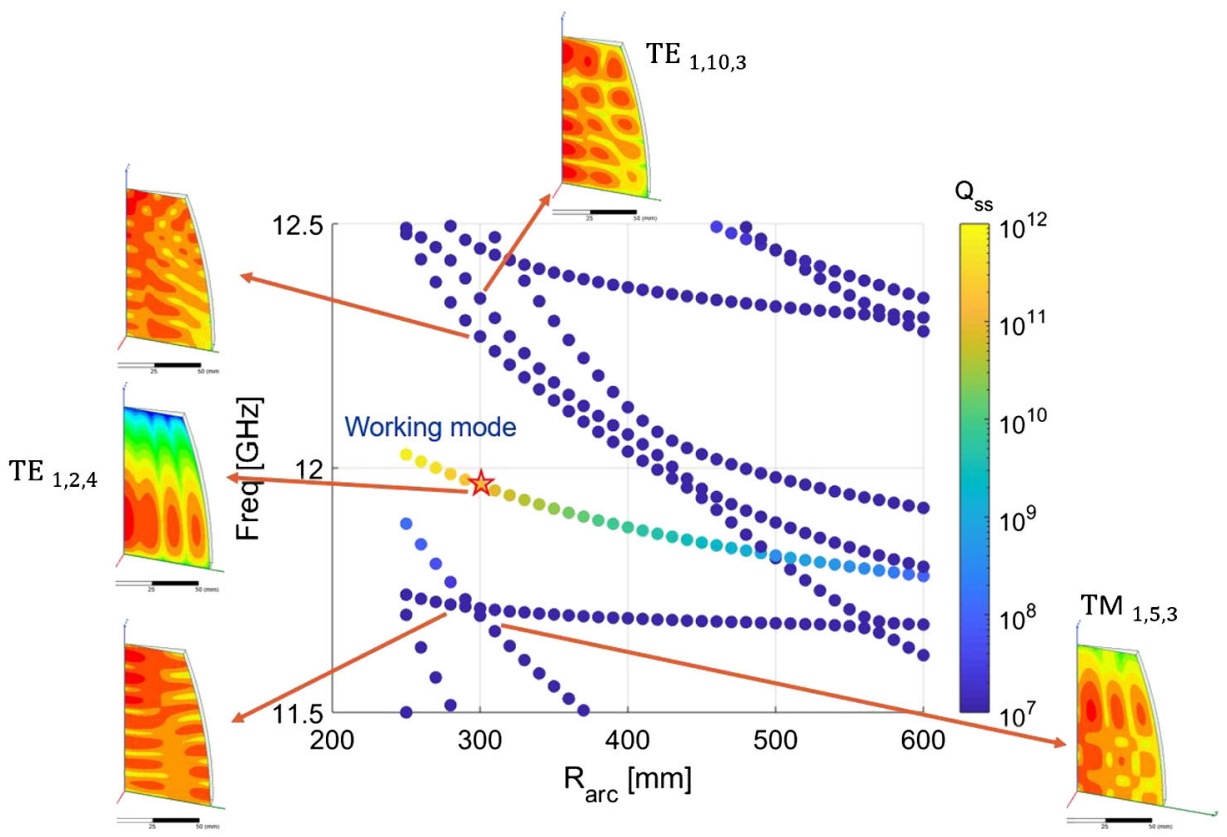

FIG. 4. Mode spectrum of $\mathrm{TE}_{1,2,4}$ mode cavity. $D Z_{\mathrm{cav}}$ is $94.8 \mathrm{~mm}$. $R_{\mathrm{cav}}$ is $49.1 \mathrm{~mm}$. Color bar represents the value of $Q_{\mathrm{ss}}$. The electrical fields of the working mode and the parasitic modes are presented in logarithmic scales. 


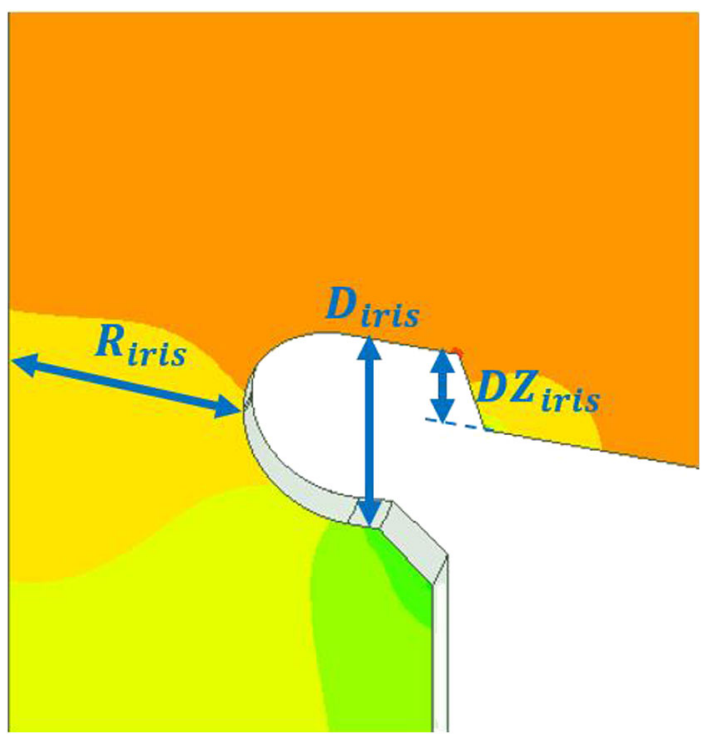

FIG. 5. Coupling iris model. The electrical field is in logarithmic scale.

The frequencies and $Q_{0}$ of the working mode and the nearest two parasitic modes are summarized in Table I. The two parasitic modes are around 248.4 and $308.4 \mathrm{MHz}$ away from the working frequency, respectively.

The electrical fields of the working mode and the parasitic modes are shown in Fig. 6.

\section{Frequency domain results}

The correction cavity model with coupling iris and circular waveguide was then simulated in the frequency domain solver of HFSS. The field distribution and $S_{11}$ frequency sweep are presented in Fig. 7.

The coupling iris between the circular waveguide and correction cavity was carefully studied and optimized to reduce the rf pulse heating $[34,35]$. To minimize the breakdown rate during operation, the dimensions were optimized to reduce the surface electromagnetic field and ensure $R_{\text {loss }}$ was lower than $1.0 \%$ [36-50]. The final coupling iris has an iris thickness $\left(D_{\text {iris }}\right)$ of $4 \mathrm{~mm}$ and a rounding radius of $1.95 \mathrm{~mm}$. As shown in Fig. 1(c), there is a frequency difference between the correction cavity and the rf signal. The resonant frequency, maximum surface electrical field, maximum surface magnetic field, and maximum modified poynting factor $\left(S_{\mathrm{c}}\right)$ [51] at the coupling iris of the six correction cavities are presented in Table II. The six correction cavities have different

TABLE I. Modes of the correction cavity.

\begin{tabular}{lcc}
\hline \hline & Frequency [GHz] & $Q_{0}$ \\
\hline Working mode & 11.9940 & 74659 \\
Parasitic mode 1 & 11.7456 & 16063 \\
Parasitic mode 2 & 12.3024 & 14700 \\
\hline \hline
\end{tabular}

$\begin{array}{llll}\text { (a) Working mode } & \text { (b) Parasitic mode1 } & \text { (c) Parasitic mode2 }\end{array}$

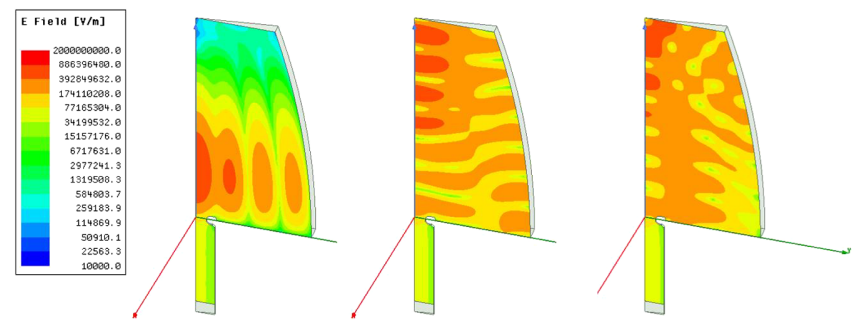

FIG. 6. Electrical fields of the working mode and the parasitic modes in the correction cavity. 8 degree slice model of the cavity with a storage energy of $1 \mathrm{~J}$ was simulated by the eigenmode solver of HFSS. The electrical fields are in logarithmic scale. (a) is the working mode at $11.994 \mathrm{GHz}$. (b) and (c) are the parasitic modes.

resonant frequencies. The fields in the table are calculated at the input power of $50 \mathrm{MW}$ at steady state in $11.994 \mathrm{GHz}$.

The pulse heating of the correction cavities are calculated at $50 \mathrm{MW}, 2250 \mathrm{~ns}$ input pulse. The temperature rise of one point on surface can be calculated as

$$
\Delta T(t)=\frac{1}{\sqrt{\pi k \rho c_{\varepsilon}}} \int_{0}^{t} \frac{R_{\mathrm{s}}\left|H\left(t^{\prime}\right)\right|^{2}}{2 \sqrt{t-t^{\prime}}} d t^{\prime}
$$

where $k=391 \mathrm{~W} /(\mathrm{mK})$ is the thermal conductivity, $\rho=$ $8.95 \times 10^{3} \mathrm{~kg} / \mathrm{m}^{3}$ is the density, and $c_{\varepsilon}=385 \mathrm{~J} /(\mathrm{kgK})$ is the specific heat capacity. $R_{\mathrm{s}}=\sqrt{\pi \mu_{0} f \rho_{\text {res }}}$ and $\rho_{\text {res }}=$ $1.7 \times 10^{-8} \Omega \mathrm{m}$ are the electric resistivities [34]. The square of the maximum surface magnetic field $\left|H_{\max }(t)\right|^{2}$ is proportional to the stored energy $W(t)$ in a resonant cavity. The stored energy in the bowl-shaped open cavity can be derived from the input and output power according to

$$
E_{\mathrm{k}}^{2}=\left(E_{\mathrm{e}}+E_{\mathrm{k}}\right)^{2}+\frac{E_{\mathrm{e}}^{2}}{\beta}+\frac{2 Q_{0} E_{\mathrm{e}}}{\omega_{0} \beta} \frac{d E_{\mathrm{e}}}{d t}
$$

where $E_{\mathrm{k}}$ is the incident electrical field from the rf source, $E_{\mathrm{e}}$ is the eletrcical field emitted from the resonant cavity, and the loaded field is $E_{1}=E_{\mathrm{k}}+E_{\mathrm{e}} . \omega_{0}$ is the angular resonant frequency and $\beta$ is the coupling factor [14]. Thus, the maximum surface magnetic field can be expressed as

$$
\left|H_{\max }(t)\right|^{2}=C_{0} W(t)
$$

$$
W(t)=\frac{Q_{0}}{2 \pi \beta f}\left(\sqrt{P_{\text {out }}(t)}-\sqrt{P_{\text {in }}(t)}\right)^{2},
$$

where $f$ is the resonant frequency, $P_{\text {out }}$ is the output power, $P_{\text {in }}$ is the input power, and $C_{0}$ was calculated by HFSS. The maximum temperature rise of the correction cavity chain at $50 \mathrm{MW}, 2250 \mathrm{~ns}$ input pulse is less than $20 \mathrm{~K}$. 
(a)

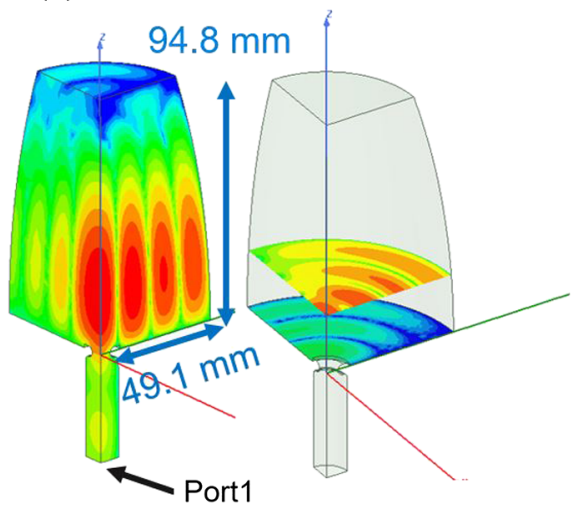

(b)

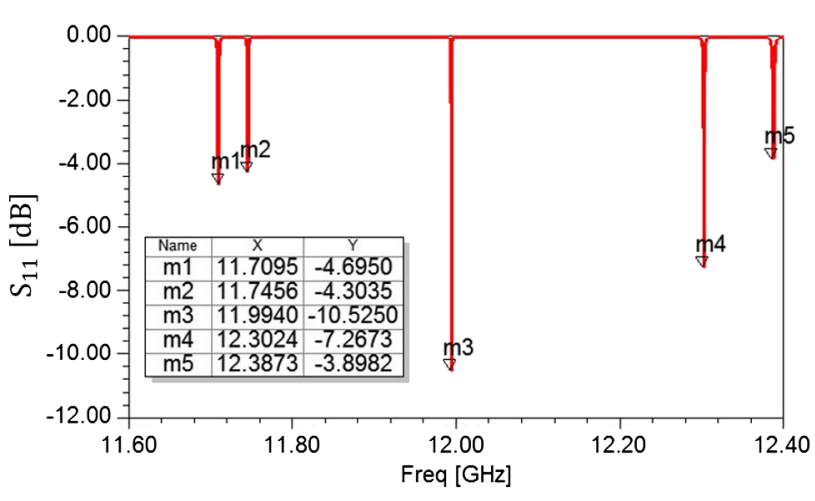

FIG. 7. (a) Electrical field of one quarter model of the correction cavity. One quarter model was simulated by the frequency domain solver of HFSS. The electrical field is in logarithmic scale. (b) $S_{11}$ frequency sweep of the correction cavity.

\section{Tolerance study}

The frequencies of the working mode and the parasitic modes are mostly determined by the geometric parameters. Micrometer mechanical tolerances have been achieved using ultraprecision diamond machining [52,53]. The analysis of the frequency sensitivity of the working mode and the nearest two parasitic modes were performed in HFSS, as summarized in Table III. It can be concluded that the most critical geometric parameter to the resonant frequency of the working mode is $R_{\text {cav }}$, so that the geometric tolerances are dominated by $R_{\text {cav. }}$. Geometric error of $0.001 \mathrm{~mm}$ which could be achieved with modern ultra high precision turning machines results in the frequency error of

TABLE II. Rf parameters of the correction cavities.

\begin{tabular}{lccc}
\hline \hline $\begin{array}{l}\text { Frequency } \\
{[\mathrm{GHz}]}\end{array}$ & $\begin{array}{c}E_{\max } \\
{[\mathrm{MV} / \mathrm{m}]}\end{array}$ & $\begin{array}{c}H_{\max } \\
{[\mathrm{kA} / \mathrm{m}]}\end{array}$ & $\begin{array}{c}S_{\mathrm{c}} \\
{\left[\mathrm{MW} / \mathrm{mm}^{2}\right]}\end{array}$ \\
\hline 11.9853 & 11.1 & 82.43 & 0.041 \\
11.9882 & 10.4 & 78.01 & 0.037 \\
11.9911 & 8.26 & 65.89 & 0.027 \\
11.9969 & 17.8 & 125.8 & 0.095 \\
11.9998 & 15.0 & 107.0 & 0.069 \\
12.0027 & 14.1 & 101.4 & 0.062 \\
\hline \hline
\end{tabular}

TABLE III. Frequency sensitivity to different geometric parameters of the working mode and the parasitic modes of the correction cavity.

\begin{tabular}{llcl}
\hline \hline & \multicolumn{3}{c}{$\Delta f / \Delta x[\mathrm{MHz} / \mathrm{mm}]$} \\
\cline { 2 - 4 } & Work & Parasitic & Parasitic \\
Dimension $\mathrm{m}$ & mode & -50.64 & -119.48 \\
\hline$R_{\text {cav }}$ & -238.45 & -96.90 & -58.06 \\
$D Z_{\text {cav }}$ & $< \pm 1.00$ & $< \pm 1.00$ & -3.43 \\
$R_{\text {arc }}$ & -1.39 & -2.82 & -3.50 \\
$R_{\text {iris }}$ & -3.38 & & \\
\hline \hline
\end{tabular}

0.24 MHz. The frequency changes of the parasitic modes caused by the machining errors on the geometry is negligible as the two parasitic modes are more than $200 \mathrm{MHz}$ away from the working mode. The whole assembly is designed to be tuning-free because the frequency error of the working mode could be compensated by the cooling water temperature with a separate chiller.

\section{E. Model with E-rotator}

The so-called E-rotator is used as $3 \mathrm{~dB}$ coupler for the SLED system of the bowl-shaped open cavity. It is an $\mathrm{rf}$ device with two rectangular and one circular waveguide ports, as shown in Fig. 8. If the input signal comes to the rectangular port 1 , the output mode is a right-circularly polarized $\mathrm{TE}_{11}$ mode, as shown in Fig. 8(a). If the input signal comes to port 2 , a left-circularly polarized $\mathrm{TE}_{11}$ mode is formed, as shown in Fig. 8(b) [30,54]. A similar (a)

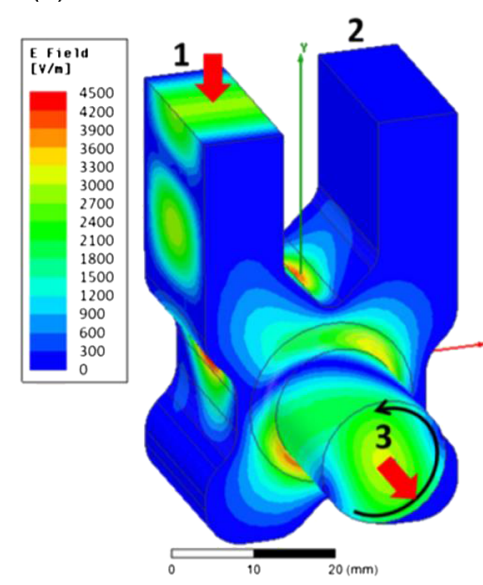

(b)

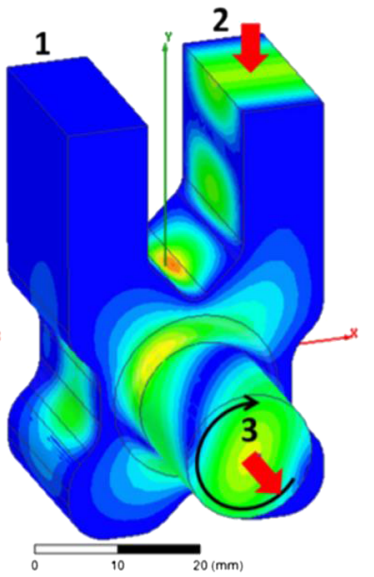

FIG. 8. E-rotator geometry and electric field distribution for $1 \mathrm{~W}$ of input power into port 1 (a) and port 2 (b). The polarization of the circular $\mathrm{TE}_{11}$ mode in the output port is indicated using black arrows [54]. 


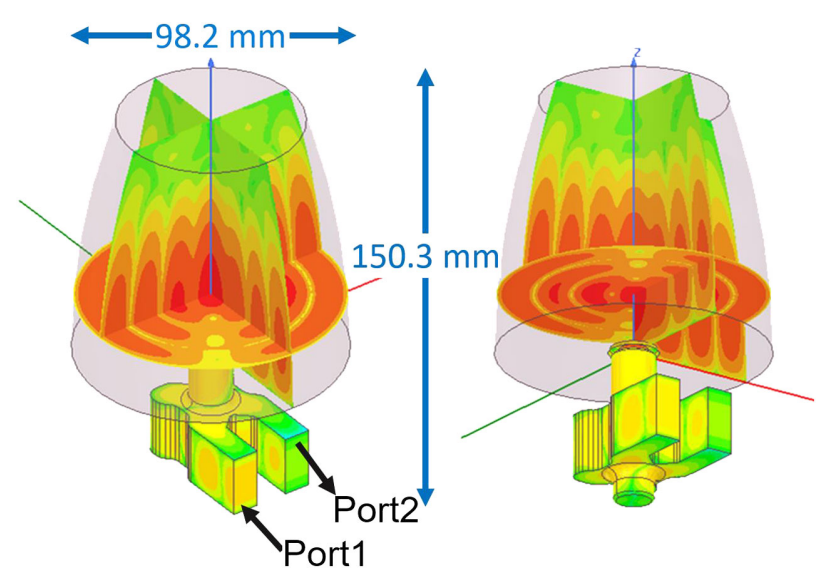

FIG. 9. Electrical field distribution of the correction cavity with the E-rotator. The electrical field is in logarithmic scale.

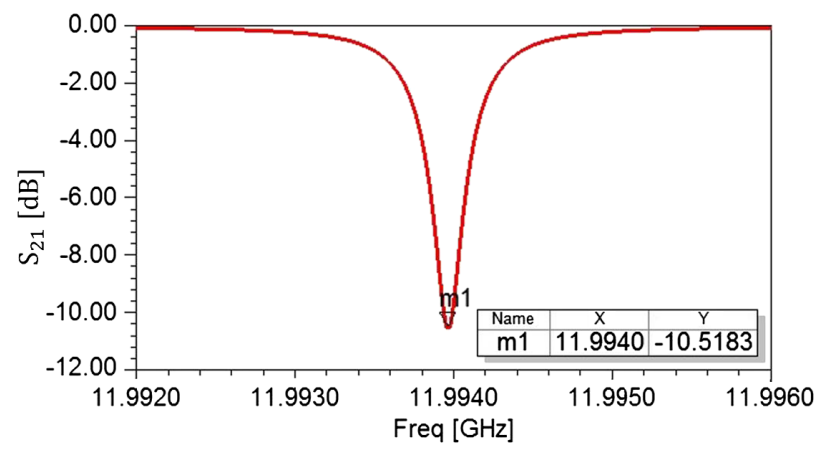

FIG. 10. $S_{21}$ frequency sweep of the correction cavity system.

device with the same functionality but different design concept, an rf polarizer, is described in Ref. [21].

The electrical field distribution of the correction cavity with the E-rotator is shown in Fig. 9. The $S_{21}$ frequency

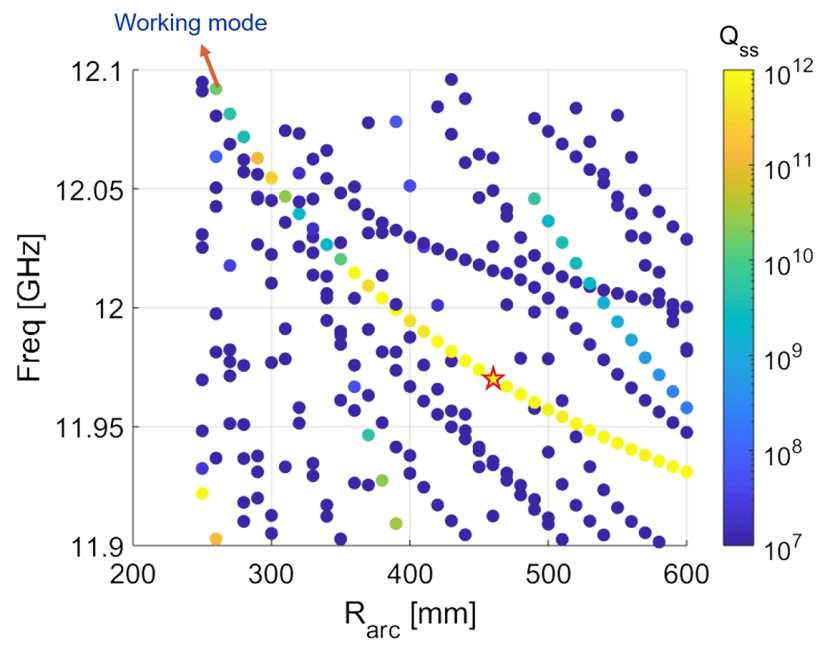

FIG. 11. Mode spectrum of $\mathrm{TE}_{1,2,13}$ mode cavity. $D Z_{\text {cav }}$ is $169.7 \mathrm{~mm}$. $R_{\text {cav }}$ is $163.1 \mathrm{~mm}$. Color bar represents the value of $Q_{\mathrm{ss}}$.
TABLE IV. Modes of the storage cavity.

\begin{tabular}{lcc}
\hline \hline & Frequency [GHz] & $Q_{0}$ \\
\hline Working mode & 11.9940 & 244542 \\
Parasitic mode 1 & 11.9635 & 51431 \\
Parasitic mode 2 & 12.0399 & 61012 \\
\hline \hline
\end{tabular}

sweep of the correction cavity system with a resonant frequency of $11.994 \mathrm{GHz}$ is shown in Fig. 10. The E-rotator has a bandwidth more than $50 \mathrm{MHz}$ which covers all the resonant frequencies of the correction cavity chain [30].

\section{STORAGE CAVITY DESIGN}

\section{A. Cavity design}

The $Q_{0}$ of the storage cavity is suggested to be $2.4 \times 10^{5}$ from Ref. [32]. $\mathrm{TE}_{1,2,13}$ mode is selected for the bowlshaped open cavity design with a $R_{\text {cav }}$ around $163 \mathrm{~mm}$. The $Q_{0}$ is around 240000 . The mode spectrum of the storage cavity for different $R_{\text {arc }} \mathrm{s}$ is shown in Fig. 11. The middle line with high $Q_{\mathrm{ss}}$ is the working mode of $\mathrm{TE}_{1,2,13}$ while the other lower $Q_{\mathrm{ss}}$ points represent the parasitic modes. $R_{\mathrm{arc}}=$ $460 \mathrm{~mm}$ is selected for the storage cavity design as it has a high $Q_{\mathrm{ss}}$ value and a large mode separation from the nearest two parasitic modes. As with the design of the correction cavity, the loss in the open boundary area is checked in the coupling iris design. The minimum $R_{\text {loss }}$ of $1.4 \%$ is obtained when $R_{\text {iris }}$ is $5.21 \mathrm{~mm}$. However, the frequency separation between working mode and parasitic modes is smaller than that of the correction cavity, as shown in Table IV. The nearest two parasitic modes are 30.5 and 45.9 $\mathrm{MHz}$ away from the working frequency, respectively. The electrical fields of the working mode and the parasitic modes are shown in Fig. 12.

\section{B. Frequency domain results}

The field distribution and $S_{11}$ frequency sweep are presented in Fig. 13. Compared with the $S_{11}$ frequency sweep of the correction cavity, the storage cavity has smaller mode separation. The transmission spectrum of
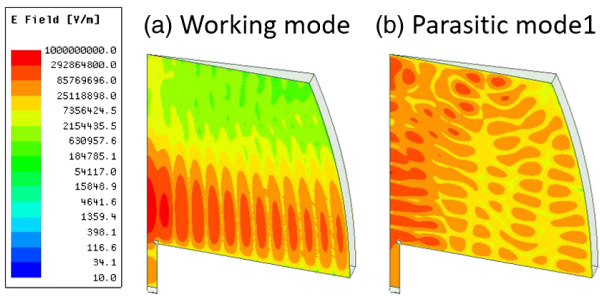

(c) Parasitic mode2

FIG. 12. Electrical field of the working mode and the parasitic modes in the storage cavity. 8 degree slice model of the cavity with a storage energy of $1 \mathrm{~J}$ was simulated by the eigenmode solver of HFSS. The electrical fields are in logarithmic scale. (a) is the working mode at $11.994 \mathrm{GHz}$. (b) and (c) are the parasitic modes. 
(a)

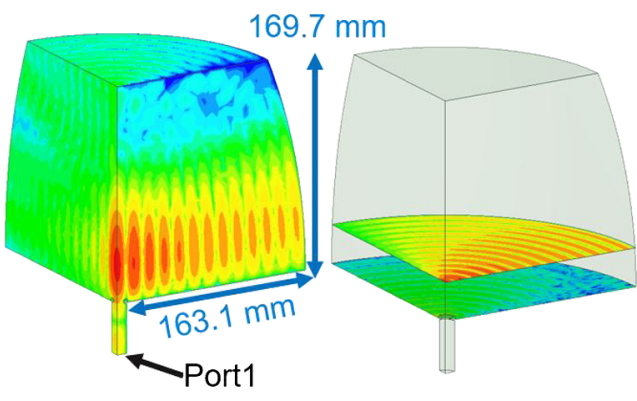

(b)

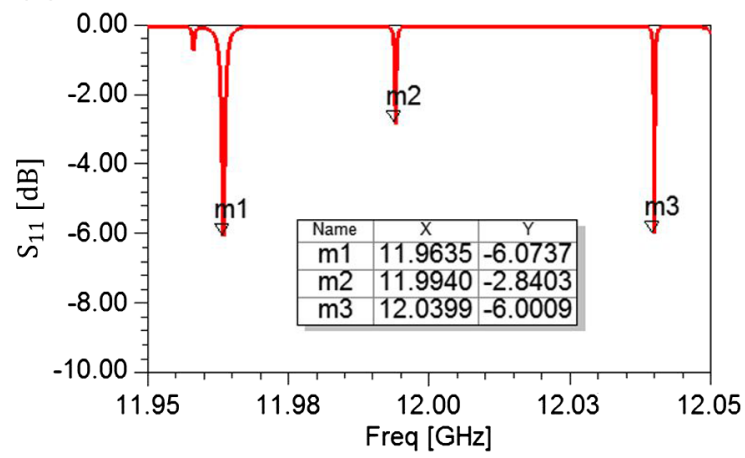

FIG. 13. (a) Electrical field of one quarter model of storage cavity. One quarter model was simulated by frequency domain solver of HFSS. The electrical field is in logarithmic scale. (b) $S_{11}$ frequency sweep of the storage cavity.

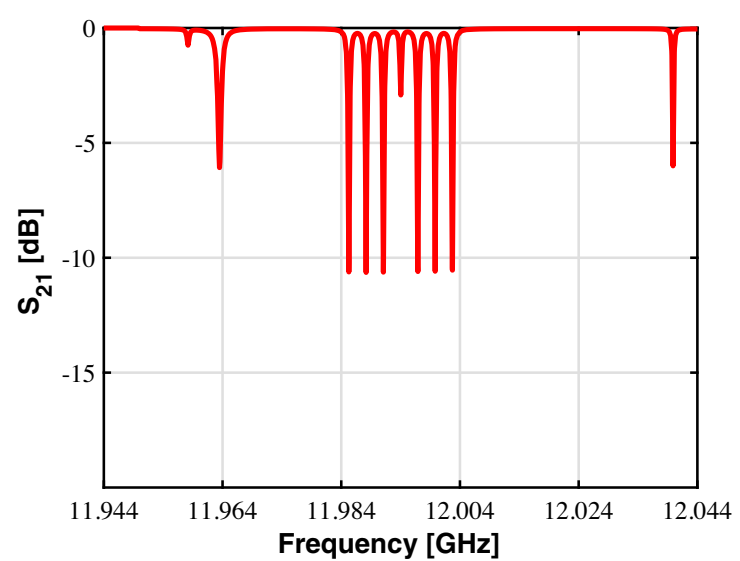

FIG. 14. Transmission spectrum of the pulse compression system based on the bowl-shape open cavities.

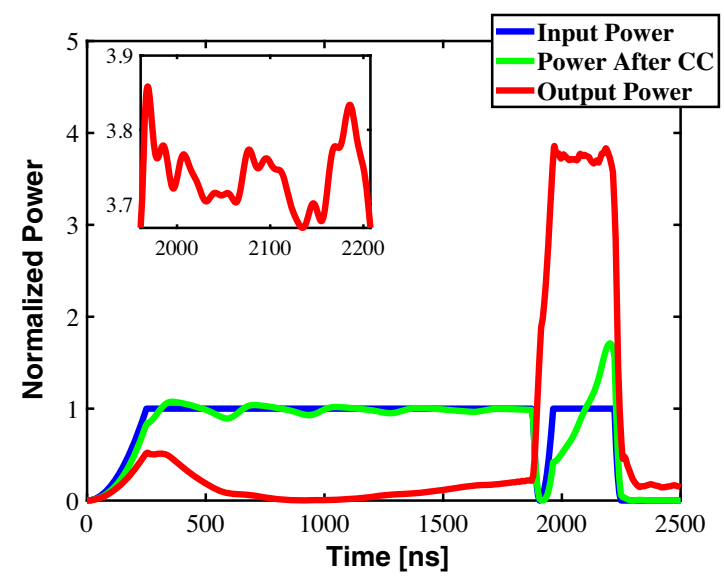

FIG. 15. Pulse shape of the pulse compression system based on the bowl-shape open cavities. The blue, green, and red curves are the input power, power after the correction cavity chain, and output power, respectively. The inset is given to show an enlarged view of output power. The $Q_{0}$ of storage cavity and coupling factor are $2.45 \times 10^{5}$ and 6.11 . The $Q_{0}$ of correction cavity and coupling factor are $7.47 \times 10^{4}$ and 1.84 . the CLIC rf pulse compression system based on the bowlshape open cavities is shown in Fig. 14. The pulse shape is shown in Fig. 15. Effect of the parasitic modes on the pulse shape is negligible.

The coupling iris between the circular waveguide and storage cavity was carefully studied and optimized to reduce the rf pulse heating. The final coupling iris has a $D_{\text {iris }}$ of $3 \mathrm{~mm}$ and a rounding radius of $1.485 \mathrm{~mm}$. The resonant frequency, maximum surface electrical field, maximum surface magnetic field, and maximum $S_{\mathrm{c}}$ at the coupling iris of the storage cavity are presented in Table V. The field in the table are calculated at an input power of $50 \mathrm{MW}$ at steady state.

As the maximum surface magnetic field of storage cavity is higher than that of correction cavities shown in Table II, the temperature rise of storage cavity was calculated at the nominal input pulse. The pulse after the correction cavity chain, compressed pulse with an $2250 \mathrm{~ns}, 50 \mathrm{MW}$ input pulse, the temperature rise, and the stored energy are shown in Fig. 16.

The maximum temperature rise of the storage cavity is 93.8 $\mathrm{K}$ at $50 \mathrm{MW}, 2250 \mathrm{~ns}$ input pulse, as shown in Fig. 16. The temperature rise is higher than that of CLIC accelerating structures at nominal power. A compact $X$-band pulse compressor described in Ref. [27] consists of a corrugated cylindrical cavity for energy storage and an rf polarizer which has same functionality as E-rotator to direct the power flow. Similar to the bowl-shaped open cavity, high-surface field is located in the iris region. The high-power tests indicated that a breakdown rate of $8.34 \times 10^{-6} 1 /$ pulse was observed at the pulse heating

TABLE V. Rf parameters of the storage cavity.

\begin{tabular}{lccc}
\hline \hline $\begin{array}{l}\text { Frequency } \\
{[\mathrm{GHz}]}\end{array}$ & $\begin{array}{c}E_{\max } \\
{[\mathrm{MV} / \mathrm{m}]}\end{array}$ & $\begin{array}{c}H_{\max } \\
{[\mathrm{kA} / \mathrm{m}]}\end{array}$ & $\begin{array}{c}S_{\mathrm{c}} \\
{\left[\mathrm{MW} / \mathrm{mm}^{2}\right]}\end{array}$ \\
\hline 11.9940 & 97.79 & 577.00 & 2.46 \\
\hline \hline
\end{tabular}




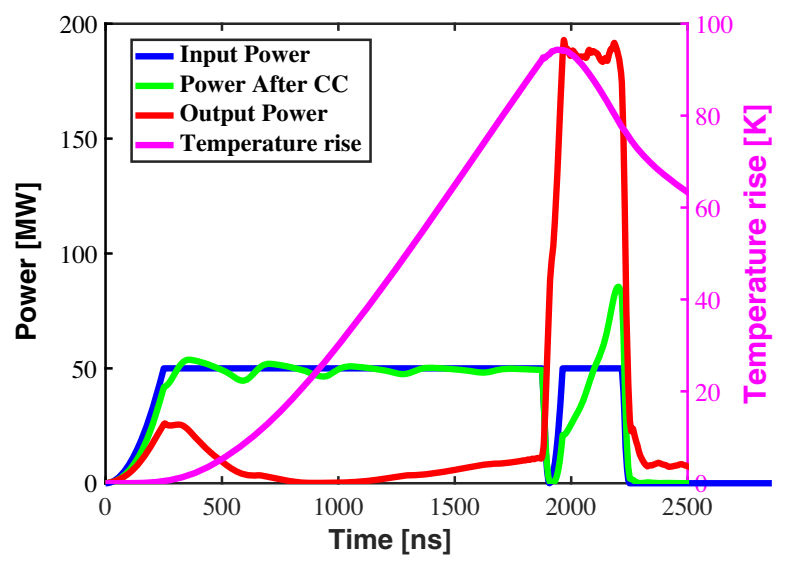

FIG. 16. Temperature rise of the storage cavity at $50 \mathrm{MW}$, $2250 \mathrm{~ns}$ input pulse. The blue, green, red, and pink are the input power, power after the correction cavity chain, output power, and temperature rise, respectively.

of $658 \mathrm{~K}$. It is speculated that bowl-shaped open cavity can work at low-breakdown rate regime because the pulse heating at iris is less than $100 \mathrm{~K}$ based on the reported experimental data.

\section{Tolerance study}

The analysis of the frequency sensitivity of the working mode and the nearest two parasitic modes were performed in HFSS, as summarized in Table VI. It can be concluded that the most critical geometric parameter to the resonant frequency of the working mode is the $R_{\mathrm{cav}}$, so that the geometric tolerances are dominated by the $R_{\text {cav }}$. Geometric error of $0.001 \mathrm{~mm}$ which could be achieved with modern ultra high precision turning machines results in the frequency error less than $0.1 \mathrm{MHz}$. This frequency error is smaller than the frequency separation between the working mode and parasitic modes. This gives us strong confidence that the mode separation will not be significantly affected by geometric errors. The whole assembly is designed to be tuning-free because the frequency error of the working mode could be easily compensated by the cooling water temperature with a separate chiller.

TABLE VI. Frequency sensitivity to different geometric parameters of the working mode and the parasitic modes of the storage cavity.

\begin{tabular}{lccc}
\hline \hline & \multicolumn{3}{c}{$\Delta f / \Delta x[\mathrm{MHz} / \mathrm{mm}]$} \\
\cline { 2 - 4 } & Work & Parasitic & Parasitic \\
Dimension $\mathrm{x}$ & mode & mode 1 & mode 2 \\
\hline$R_{\text {cav }}$ & -72.66 & -24.41 & -34.07 \\
$D Z_{\text {cav }}$ & $< \pm 1.00$ & -45.50 & -37.64 \\
$R_{\text {arc }}$ & $< \pm 1.00$ & $< \pm 1.00$ & $< \pm 1.00$ \\
$R_{\text {iris }}$ & $< \pm 1.00$ & -2.44 & $< \pm 1.00$ \\
\hline \hline
\end{tabular}

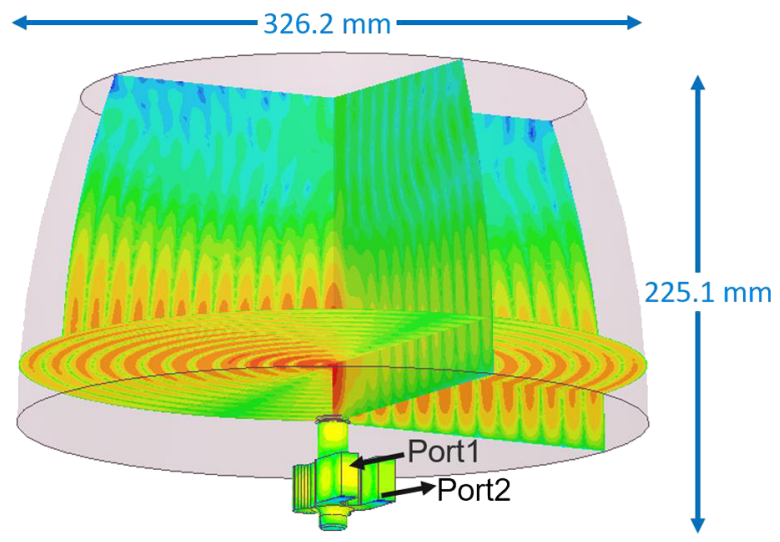

FIG. 17. Electrical field distribution of the storage cavity with the E-rotator. The electrical field is in logarithmic scale.

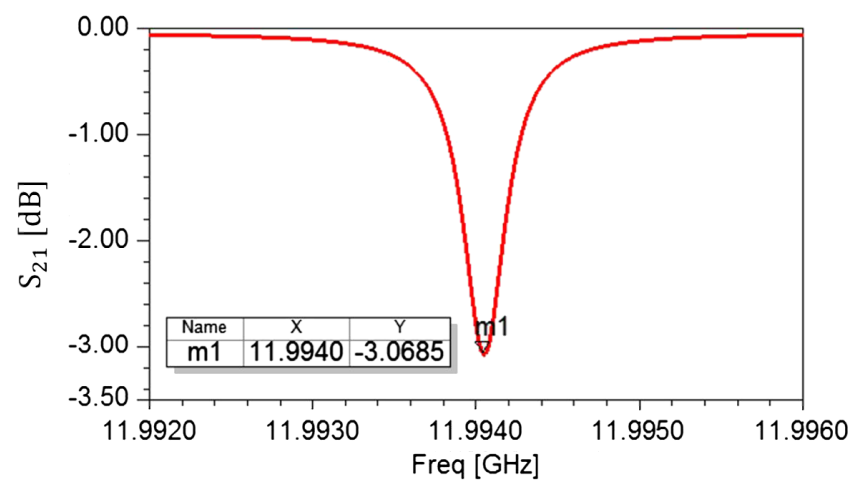

FIG. 18. $S_{21}$ frequency sweep of the storage cavity system.

\section{Model with E-rotator}

The electrical field distribution of the storage cavity with the E-rotator is shown in Fig. 17. The $S_{21}$ frequency sweep of the storage cavity system is shown in Fig. 18.

\section{CONCLUSION}

A new $X$-band high-power rotating mode SLED-type rf pulse compressor is proposed. It is based on a single open bowl-shaped energy storage cavity working at quasispherical rotating mode. It has high $Q_{0}$ and compact size. The cavity can be fabricated by lathe with high accuracy due to its symmetric shape. Tolerance study indicates that the frequency shift caused by the machining error can be easily compensated by the water temperature. The cavity is coupled to a circular waveguide with a compact rotating mode launcher. An open cavity working at $\mathrm{TE}_{1,2,4}$ mode is proposed for the correction cavity design of CLIC rf pulse compression system. It has a $Q_{0}$ of 74659 and a diameter less than $5 \mathrm{~cm}$. $\mathrm{TE}_{1,2,13}$ mode is proposed for the storage cavity design of CLIC rf pulse compression system. It has a high $Q_{0}$ of 244542 and a diameter less than $33 \mathrm{~cm}$. 


\section{ACKNOWLEDGMENTS}

The authors would like to thank Dr. Igor Syratchev, Dr. Walter Wuensch, and Dr. Lee Millar for their helpful discussions and suggestions on this work.

[1] M. Aicheler, P. Burrows, N. C. Lasheras, R. Corsini, M. Draper, J. Osborne, D. Schulte, S. Stapnes, and M. Stuart (CLIC Accelerator Collaboration), The Compact Linear Collider (CLIC)_Project Implementation Plan, edited by M. Aicheler, CERN Yellow Reports: Monographs (2019), p. 247, https://doi.org/10.23731/CYRM-2018-004.

[2] S. Y. Kazakov, Pulse shape correction for rf pulse compression system, in Proceedings of the 3rd European Particle Accelerator Conference (EPAC'92), Berlin, Germany, 1992 (JACOW, Geneva, Switzerland, 1992), pp. 1247-1248.

[3] P. Wang, H. Zha, I. Syratchev, J. Shi, and H. Chen, rf design of a pulse compressor with correction cavity chain for klystron-based compact linear collider, Phys. Rev. Accel. Beams 20, 112001 (2017).

[4] Y. Jiang, H. Zha, P. Wang, J. Shi, H. Chen, W. L. Millar, and I. Syratchev, Demonstration of a cavity-based pulse compression system for pulse shape correction, Phys. Rev. Accel. Beams 22, 082001 (2019).

[5] R. Akre, V. Bharadwaj, P. Emma, and P. Krejcik, SLAC linac RF performance for LCLS, arXiv:physics/0008171.

[6] T. Schietinger, Commissioning experience and beam physics measurements at the SwissFEL injector test facility, Phys. Rev. Accel. Beams 19, 100702 (2016).

[7] T. Inagaki, C. Kondo, H. Maesaka, T. Ohshima, Y. Otake, T. Sakurai, K. Shirasawa, and T. Shintake, High-gradient $C$-band linac for a compact x-ray free-electron laser facility, Phys. Rev. ST Accel. Beams 17, 080702 (2014).

[8] Y. Joo, H.-S. Lee, W. Hwang, Y. Park, K. Oh, and B.-J. Lee, Design study of a new sled system with a biplanar 3-db power divider and dual side-wall coupling-irises for the pal xfel, J. Korean Phys. Soc. 63, 1253 (2013).

[9] W. Fang, Q. Gu, X. Sheng, C. Wang, D. Tong, L. Chen, S. Zhong, J. Tan, G. Lin, Z. Chen, and Z. Zhao, Design, fabrication and first beam tests of the $C$-band RF acceleration unit at SINAP, Nucl. Instrum. Methods Phys. Res., Sect. A 823, 91 (2016).

[10] M. Diomede, D. Alesini, M. Bellaveglia, B. Buonomo, F. Cardelli, N. C. Lasheras, E. Chiadroni, G. Di Pirro, M. Ferrario, A. Gallo, A. Ghigo, A. Giribono, A. Grudiev, L. Piersanti, B. Spataro, C. Vaccarezza, and W. Wuensch, Preliminary RF design of an $X$-band linac for the EuPRAXIA@SPARC LAB project, Nucl. Instrum. Methods Phys. Res., Sect. A 909, 243 (2018).

[11] B. Woolley, G. Burt, A. C. Dexter, R. Peacock, W. L. Millar, N. C. Lasheras, A. Degiovanni, A. Grudiev, G. Mcmonagle, I. Syratchev, W. Wuensch, E. R. Castro, and J. G. Navarro, High-gradient behavior of a dipole-mode if structure, Phys. Rev. Accel. Beams 23, 122002 (2020).

[12] B. Woolley, I. Syratchev, and A. Dexter, Control and performance improvements of a pulse compressor in use for testing accelerating structures at high power, Phys. Rev. Accel. Beams 20, 101001 (2017).

[13] J. Liu, J. Shi, H. Zha, A. Grudiev, P. Wang, Y. Du, and H. Chen, Analytic RF design of a linear accelerator with a SLED-I type RF pulse compressor, Nucl. Sci. Tech. 31, 107 (2020).

[14] Z. D. Farkas, H. A. Hoag, G. A. Loew, and P. B. Wilson, SLED: A method of doubling SLAC's energy, in Proceedings of the 9th International Conference on High-Energy Accelerators, SLAC, CA, USA, 1974 (A.E.C., Washington, DC, 1975), pp. 576-583.

[15] V. Balakin and I. Syrachev, Status VLEPP rf power multiplier (VPM), in Proceedings of the 3rd European Particle Accelerator Conference (EPAC'92), Berlin, Germany, 1992 (JACOW, Geneva, Switzerland, 1992), pp. 1173-1175.

[16] S. G. Tantawi, C. D. Nantista, V. A. Dolgashev, C. Pearson, J. Nelson, K. Jobe, J. Chan, K. Fant, J. Frisch, and D. Atkinson, High-power multimode $X$-band rf pulse compression system for future linear colliders, Phys. Rev. ST Accel. Beams 8, 042002 (2005).

[17] N. Akasaka, M. Tejima, I. Watanabe, T. Takeshita, T. Tauchi, H. Mizuno, J. Fujimoto, T. Kon, C. Suzuki, K. Matsukado et al., JLC design study, Technical Report No. 97-1, KEK, 1997.

[18] I. Syrachev, The Progress of $X$-Band "Open" Cavity RF Pulse Compression Systems, in Proceedings of the 4rd European Particle Accelerator Conference (EPAC'94), London, United Kingdom, 1994 (JACOW, Geneva, Switzerland, 1994), pp. 375-379.

[19] P. B. Wilson, Z. D. Farkas, and R. D. Ruth, SLED II: A new method of rf pulse compression, in 15th Linear Accelerator Conference (LINAC'1990), Albuquerque, NM, USA, Sep 10-14, 1990 (JACOW, Geneva, Switzerland, 1990), pp. 204-206.

[20] J. W. Wang, S. G. Tantawi, C. Xu, M. Franzi, P. Krejcik, G. Bowden, S. Condamoor, Y. Ding, V. Dolgashev, J. Eichner, A. Haase, J. R. Lewandowski, and L. Xiao, Development for a supercompact $X$-band pulse compression system and its application at SLAC, Phys. Rev. Accel. Beams 20, 110401 (2017).

[21] M. Franzi, J. Wang, V. Dolgashev, and S. Tantawi, Compact rf polarizer and its application to pulse compression systems, Phys. Rev. Accel. Beams 19, 062002 (2016).

[22] C. Xu, S. Tantawi, and J. Wang, Conceptual design of $X$ band waveguide dual circular polarizer, Phys. Rev. Accel. Beams 19, 062003 (2016).

[23] Z. Li, W. Fang, Q. Gu, and Z. Zhao, RF design of a $C$-band compact spherical RF pulse compressor for SXFEL, Nucl. Instrum. Methods Phys. Res., Sect. A 863, 7 (2017).

[24] J. Lei, X. He, M. Hou, G. Shu, G. Pei, X. Li, and H. Wang, RF design of an $S$-band spherical cavity pulse compressor, Radiat. Detect. Technol. Methods 1, 16 (2017).

[25] P. Wang, J. Shi, H. Zha, D. Cao, M. Peng, Z. Liu, C. Cheng, and H. Chen, Development of an $S$-band spherical pulse compressor, Nucl. Instrum. Methods Phys. Res., Sect. A 901, 84 (2018).

[26] Z. Li, W. Fang, Q. Gu, J. Tan, X. Huang, and Z.-T. Zhao, Design, fabrication, and cold-test results of $C$-band spherical RF pulse compressor prototype, Radiat. Detect. Technol. Methods 3, 21 (2019). 
[27] Y. Jiang, H. Zha, J. Shi, M. Peng, X. Lin, and H. Chen, A Compact $X$-Band Microwave Pulse Compressor Using a Corrugated Cylindrical Cavity, IEEE Trans. Microwave Theory Tech. 69, 1586 (2021).

[28] G. Burt, S. Samsonov, A. Phelps, V. Bratman, K. Ronald, G. Denisov, W. He, A. Young, A. Cross, and I. Konoplev, Microwave pulse compression using a helically corrugated waveguide, IEEE Trans. Plasma Sci. 33, 661 (2005).

[29] L. Zhang, S. V. Mishakin, W. He, S. V. Samsonov, M. McStravick, G. G. Denisov, A. W. Cross, V. L. Bratman, C. G. Whyte, C. W. Robertson, A. R. Young, K. Ronald, and A.D. R. Phelps, Experimental Study of Microwave Pulse Compression Using a Five-Fold Helically Corrugated Waveguide, IEEE Trans. Microwave Theory Tech. 63, 1090 (2015).

[30] A. Grudiev, Design of compact high power rf components at $X$-band, Report No. CLIC-Note-1067, CERN, Geneva, 2016.

[31] J. Liu and A. Grudiev, RF design of accelerating structure for the main linac of the klystron-based first stage of CLIC at $380 \mathrm{GeV}$, Report No. CLIC-Note-1082, CERN, Geneva, 2018.

[32] J. Cai and I. Syratchev, The design update of the $X$-band RF pulse compressor with Correction Cavities for the CLIC $380 \mathrm{GeV}$ klystron based accelerator, Report No. CLIC-Note-1166, CERN, Geneva, 2020.

[33] HFSS, https://www.ansys.com/products/electronics/ansyshfss.

[34] D. P. Pritzkau and R. H. Siemann, Experimental study of rf pulsed heating on oxygen free electronic copper, Phys. Rev. ST Accel. Beams 5, 112002 (2002).

[35] L. Laurent, S. Tantawi, V. Dolgashev, C. Nantista, Y. Higashi, M. Aicheler, S. Heikkinen, and W. Wuensch, Experimental study of rf pulsed heating, Phys. Rev. ST Accel. Beams 14, 041001 (2011).

[36] A. Saressalo, I. Profatilova, W. L. Millar, A. Kyritsakis, S. Calatroni, W. Wuensch, and F. Djurabekova, Effect of dc voltage pulsing on high-vacuum electrical breakdowns near cu surfaces, Phys. Rev. Accel. Beams 23, 113101 (2020).

[37] I. Profatilova, X. Stragier, S. Calatroni, A. Kandratsyeu, E. R. Castro, and W. Wuensch, Breakdown localisation in a pulsed DC electrode system, Nucl. Instrum. Methods Phys. Res., Sect. A 953, 163079 (2020).

[38] M. Jacewicz, J. Eriksson, R. Ruber, S. Calatroni, I. Profatilova, and W. Wuensch, Temperature-Dependent Field Emission and Breakdown Measurements Using a Pulsed High-Voltage Cryosystem, Phys. Rev. Applied 14, 061002 (2020).

[39] I. V. Uimanov, D. L. Shmelev, and S. A. Barengolts, Effect of electrode temperature on radiofrequency vacuum breakdown characteristics, J. Phys. D 54, 065205 (2021).

[40] A. Vnuchenko, D. E. Pereira, B. G. Martinez, S. Benedetti, N. C. Lasheras, M. Garlasch, A. Grudiev, G. McMonagle, S. Pitman, I. Syratchev, M. Timmins, R. Wegner, B. Woolley, W. Wuensch, and A. F. Golfe, High-gradient testing of an $S$ band, normal-conducting low phase velocity accelerating structure, Phys. Rev. Accel. Beams 23, 084801 (2020).

[41] M. A. K. Othman, J. Picard, S. Schaub, V. A. Dolgashev, S. M. Lewis, J. Neilson, A. Haase, S. Jawla, B. Spataro, R. J. Temkin, S. Tantawi, and E. A. Nanni, Experimental demonstration of externally driven millimeter-wave particle accelerator structure, Appl. Phys. Lett. 117, 073502 (2020).

[42] E. Z. Engelberg, A. B. Yashar, Y. Ashkenazy, M. Assaf, and I. Popov, Theory of electric field breakdown nucleation due to mobile dislocations, Phys. Rev. Accel. Beams 22, 083501 (2019).

[43] S. A. Barengolts, I. V. Uimanov, and D. L. Shmelev, Prebreakdown Processes in a metal surface microprotrusion exposed to an RF electromagnetic field, IEEE Trans. Plasma Sci. 47, 3400 (2019).

[44] S. A. Barengolts, V. G. Mesyats, V. I. Oreshkin, E. V. Oreshkin, K. V. Khishchenko, I. V. Uimanov, and M. M. Tsventoukh, Mechanism of vacuum breakdown in radiofrequency accelerating structures, Phys. Rev. Accel. Beams 21, 061004 (2018).

[45] D. Alesini, A. Battisti, M. Bellaveglia, F. Cardelli, A. Falone, A. Gallo, V. Lollo, D. T. Palmer, L. Pellegrino, L. Piersanti, S. Pioli, A. Variola, V. Pettinacci, and L. Palumbo, Design, realization, and high power test of high gradient, high repetition rate brazing-free $S$-band photogun, Phys. Rev. Accel. Beams 21, 112001 (2018).

[46] D. Alesini, M. Bellaveglia, F. Cardelli, R. Di Raddo, A. Gallo, V. Lollo, L. Piersanti, A. Variola, L. Palumbo, F. Poletto, and P. Favaron, Realization and high power test of damped $C$-band accelerating structures, Phys. Rev. Accel. Beams 23, 042001 (2020).

[47] J. Shao, S. P. Antipov, S. V. Baryshev, H. Chen, M. Conde, D. S. Doran, W. Gai, C. Jing, W. Liu, J. Power, J. Qiu, J. Shi, D. Wang, F. Wang, C. E. Whiteford, E. Wisniewski, and L. Xiao, Observation of Field-Emission Dependence on Stored Energy, Phys. Rev. Lett. 115, 264802 (2015).

[48] F. Wang, C. Adolphsen, and C. Nantista, Performance limiting effects in $X$-band accelerators, Phys. Rev. ST Accel. Beams 14, 010401 (2011).

[49] X. Wu, J. Shi, H. Chen, J. Shao, T. Abe, T. Higo, S. Matsumoto, and W. Wuensch, High-gradient breakdown studies of an $X$-band Compact Linear Collider prototype structure, Phys. Rev. Accel. Beams 20, 052001 (2017).

[50] X. Wu, H. Zha, J. Shi, H. Chen, T. Abe, T. Higo, and S. Matsumoto, Design, fabrication, and high-gradient testing of $X$-band choke-mode damped structures, Phys. Rev. Accel. Beams 22, 031001 (2019).

[51] A. Grudiev, S. Calatroni, and W. Wuensch, New local field quantity describing the high gradient limit of accelerating structures, Phys. Rev. ST Accel. Beams 12, 102001 (2009).

[52] J. Liu, J. Shi, A. Grudiev, H. Zha, and H. Chen, Tolerance study of travelling-wave accelerating structure for the main linac of the klystron-based firststage of Compact LinearCollider, Nucl. Instrum. Methods Phys. Res., Sect. A 981, 164499 (2020).

[53] J. Sauza-Bedolla, H. Bursali, N. C. Lasheras, A. Grudiev, S. Lebet, E. Rodriguez-Castro, P. Sobrino-Mompean, A. Solodko, and K. Szypula, Preserving micron tolerances through the assembly process of an $X$-band accelerating structure, in Proceedings of the 29th Linear Accelerator Conference (LINAC'2018), Beijing, China, 2018 (JACOW, Geneva, Switzerland, 2018), pp. 377-379.

[54] P. Craievich et al., Novel $X$-band transverse deflection structure with variable polarization, Phys. Rev. Accel. Beams 23, 112001 (2020). 